Check for updates

Cite this: J. Mater. Chem. C, 2018, 6, 2502

Received 5th September 2017, Accepted 30th January 2018

DOI: $10.1039 / c 7 t c 04070 a$

rsc.li/materials-c

\title{
Polystyrene nanoparticle-templated hollow titania nanosphere monolayers as ordered scaffolds $\dagger$
}

\author{
V. Robbiano, ${ }^{a}$ G. M. Paternò, ${ }^{a}$ G. F. Cotella, ${ }^{a}$ T. Fiore, ${ }^{b}{ }^{b}$ M. Dianetti, \\ M. Scopelliti, (D) ${ }^{\text {b }}$ F. Brunetti, ${ }^{c}$ B. Pignataro (D) bd and F. Cacialli (D)*a
}

\begin{abstract}
We report a novel multi-step method for the preparation of ordered mesoporous titania scaffolds and show an illustrative example of their application to solar cells. The method is based on (monolayer) colloidal nanosphere lithography that makes use of polystyrene nanoparticles organised at a water-air interface and subsequently transferred onto a solid substrate. A titania precursor solution (titanium(Iv) isopropoxide in ethanol) is then drop-cast onto the monolayer and left to "incubate" overnight. Surprisingly, instead of the expected inverse monolayer-structure, a subsequent calcination step of the precursor yields an ordered monolayer of hollow titania nanospheres with a wall thickness of 30-50 nm, and a slightly larger diameter than that of the starting spheres. X-ray diffraction (XRD) and $X$-ray photoelectron spectroscopy (XPS) characterization of such scaffolds confirm that they consist of nanocrystalline anatase titania, and that any polystyrene/carbon residues in the scaffolds are below the XPS detection level. As an illustrative application we prepared perovskite solar cells incorporating the templated-nanoparticle scaffolds displaying a respectable power conversion efficiency of $\sim 9 \%$, twice as large as that of our unoptimized "reference" cells (i.e. incorporating conventional mesoporous or compact titania scaffolds), thereby also demonstrating that the process is relatively robust with respect to optimization of the process parameters.
\end{abstract}

\section{Introduction}

Thanks to its wide bandgap (3.7 eV) and its relatively high electron affinity $(\sim 5 \mathrm{eV}),{ }^{1}$ titanium dioxide (titania, or $\left.\mathrm{TiO}_{2}\right)$ has found crucial application in a variety of photovoltaic devices from the so-called dye-sensitised solar cells, to the more recent organo-halide perovskite solar cells, ${ }^{2,3}$ as well as in photocatalysis. ${ }^{4}$ In such applications, the porosity and meso/ nanostructural arrangement play an important role in charge transport processes that follow the primary photoexcitation and exciton splitting events. Even though various other oxides have also been investigated, ${ }^{5-8} \mathrm{TiO}_{2}$ remains the most extensively used scaffold material. ${ }^{3,9}$

\footnotetext{
${ }^{a}$ Department of Physics and Astronomy and London Centre for Nanotechnology, University College London, WC1E 6BT, UK. E-mail: f.cacialli@ucl.ac.uk

${ }^{b}$ Dipartimento di Fisica e Chimica (DiFC), Università degli Studi di Palermo, V.le delle Scienze, Ed. 17 - 90128, Palermo, Italy

${ }^{c}$ CHOSE Centre for Hybrid and Organic Solar Energy, Department of Electronic Engineering, University of Rome Tor Vergata, Via del Politecnico 1, 00133 Rome, Italy

${ }^{d}$ Aten Center, Università degli Studi di Palermo, V.le delle Scienze, Ed. 18 - 90128 Palermo, Italy

$\dagger$ Electronic supplementary information (ESI) available: AFM images and analysis, SEM images, XRD spectra, forward and reverse $J-V$ characteristics, Kelvin probe measurements and fitting of the $J-V$ curves. See DOI: 10.1039/c7tc04070a
}

Interestingly, photonic structuring for photon recycling/ light trapping is also a current area of interest in photovoltaics, with a view to optimizing the optical design for overall absorption, but there have been only a few studies aimed at templating mesoporous titania films onto well-ordered monolayers and/or reporting hollow structures. In one case ${ }^{10}$ ordered structures were only reported for (silica) hemispheres and not for full spheres. Three-dimensional titania inverse opals (not monolayers) were also reported ${ }^{11}$ although these structures are typically much thicker and allow poor control over the overall thickness of the mesoporous layer. Other methods ${ }^{12-16}$ have been reported in the literature to produce either random or hollow spheres (e.g. by using micelles, ${ }^{16}$ etc.) but not in an ordered fashion. ${ }^{17}$ The advantage of ordered structures is that not only do they offer the intrinsic properties of the material, but also afford properties arising from the periodic ordering.

Here we show the possibility of obtaining hexagonallyordered hollow (hh) "essentially complete" spheres, by simply starting from a monolayer template of the polystyrene beads and by replacing the spin-coating of the titania precursor with drop-casting and subsequent (overnight) incubation of the same precursor solution, followed by multi-step calcination. As an illustrative example of a possible application, we further integrate such scaffolds into organo lead-halide perovskite solar cells. Although we have not optimised the preparation 
of our perovskite solar cells (both for the reference and for the templated structures), which are then somewhat lower than the state-of-the-art, interestingly, we find that our templated structures show higher efficiency compared to standard cells prepared onto our mesoporous or compact titania layers (albeit unoptimized), and demonstrate that the process is robust with respect to optimization of the process parameters.

\section{Experimental detail}

\section{Self-assembled monolayer materials and fabrication}

Commercial polystyrene (PS) monodisperse nanospheres (10\% in volume, diameter $a=370$ and $430 \mathrm{~nm}$; refractive index, $n_{\mathrm{PS}}=1.59$; standard deviation $<5 \%$ ) water suspensions (Thermo Scientific) were used for the preparation of monolayers. These were prepared via the self-assembling method at the air-water interface. Nanosphere suspensions were properly diluted in ethanol with a $1: 2$ ratio and then we added $200 \mu \mathrm{L}$ of the suspension dropwise using a calibrated micropipette onto the surface of the water contained inside a reservoir (e.g. a Petri dish). The resulting self-assembled ordered two-dimensional hexagonal-closed packed structures were transferred onto fluorinated tin-oxide (FTO)/compact $\mathrm{TiO}_{2}\left(\mathrm{c}-\mathrm{TiO}_{2}\right)$ substrates dipped into the reservoir. The good quality of the PS nanosphere monolayers and the effectiveness of the transfer process are confirmed by AFM characterization (Fig. 1(b and c)).

To obtain the titania nanostructures, the nanosphere monolayers were infiltrated with the titania precursor solution. This was prepared by mixing $100 \mu \mathrm{L}$ of liquid titanium(Iv) isopropoxide (Sigma Aldrich, 97\%) with $10.0 \mathrm{~mL}$ of ethanol and $2 \mu \mathrm{L}$ of
$\mathrm{HCl}(2 \mathrm{M})$. One droplet of the solution was carefully deposited onto the samples that were then left to dry at room temperature for $12 \mathrm{~h}$. Eventually, the monolayers were calcined via multiple step annealing in a furnace (fast heating from room temperature to $150{ }^{\circ} \mathrm{C}$, approx. $30{ }^{\circ} \mathrm{C} \mathrm{min}{ }^{-1}$, then to $300{ }^{\circ} \mathrm{C}$ with a rate of $5{ }^{\circ} \mathrm{C} \min ^{-1}$ and left for 2 hours, finally increased again at a rate of $5{ }^{\circ} \mathrm{C} \min ^{-1}$ to $500{ }^{\circ} \mathrm{C}$ and left for 2 hours) to obtain a hexagonal close-packed titania nanosphere layer.

\section{Solar cell materials and fabrication}

To form the desired electrode pattern, FTO/glass substrates (Sigma Aldrich, $13 \Omega \square^{-1}, 15 \mathrm{~mm} \times 20 \mathrm{~mm}$ ) were patterned with zinc powder and $2 \mathrm{M} \mathrm{HCl}$ diluted in deionized water. The obtained substrates were first cleaned with acetone and isopropanol in an ultrasonic bath (12 min each step), then treated with an oxygen plasma for $10 \mathrm{~min}$ to remove any remaining organic residues. ${ }^{18}$ The $\mathrm{c}^{-\mathrm{TiO}_{2}}$ film was deposited by spincoating a solution of $350 \mu \mathrm{L}$ of titanium(Iv) isopropoxide and $5.8 \mu \mathrm{L}$ of $\mathrm{HCl}$ (Sigma Aldrich, reagent grade 37\%) diluted in $5 \mathrm{~mL}$ of ethanol at $2000 \mathrm{rpm}$ for $60 \mathrm{~s}$, with subsequent sintering on a hot plate at $500{ }^{\circ} \mathrm{C}$ for $30 \mathrm{~min}$ in an ambient atmosphere. For the reference devices, a $\mathrm{TiO}_{2}$ paste (nanocrystals with an average size of $\sim 20 \mathrm{~nm}$, Dyesol 18NR-T) was diluted to $1: 5$ parts with ethanol, then spin-coated on the substrates at $1000 \mathrm{rpm}$ for $60 \mathrm{~s}$ (thickness $\sim 300 \mathrm{~nm}$ ), and finally sintered at $500{ }^{\circ} \mathrm{C}$ for 30 min to obtain a mesoporous scaffold layer.

After the deposition of the titania nanostructured or mesoporous films, the perovskite layer was obtained by spin-coating the $\mathrm{CH}_{3} \mathrm{NH}_{3} \mathrm{PbI}_{3-x} \mathrm{Cl}_{x}$ solution (Ossila Ltd) in a nitrogen atmosphere at $2500 \mathrm{rpm}$ for $60 \mathrm{~s}$ and by heating at $90{ }^{\circ} \mathrm{C}$ for $90 \mathrm{~min}$.

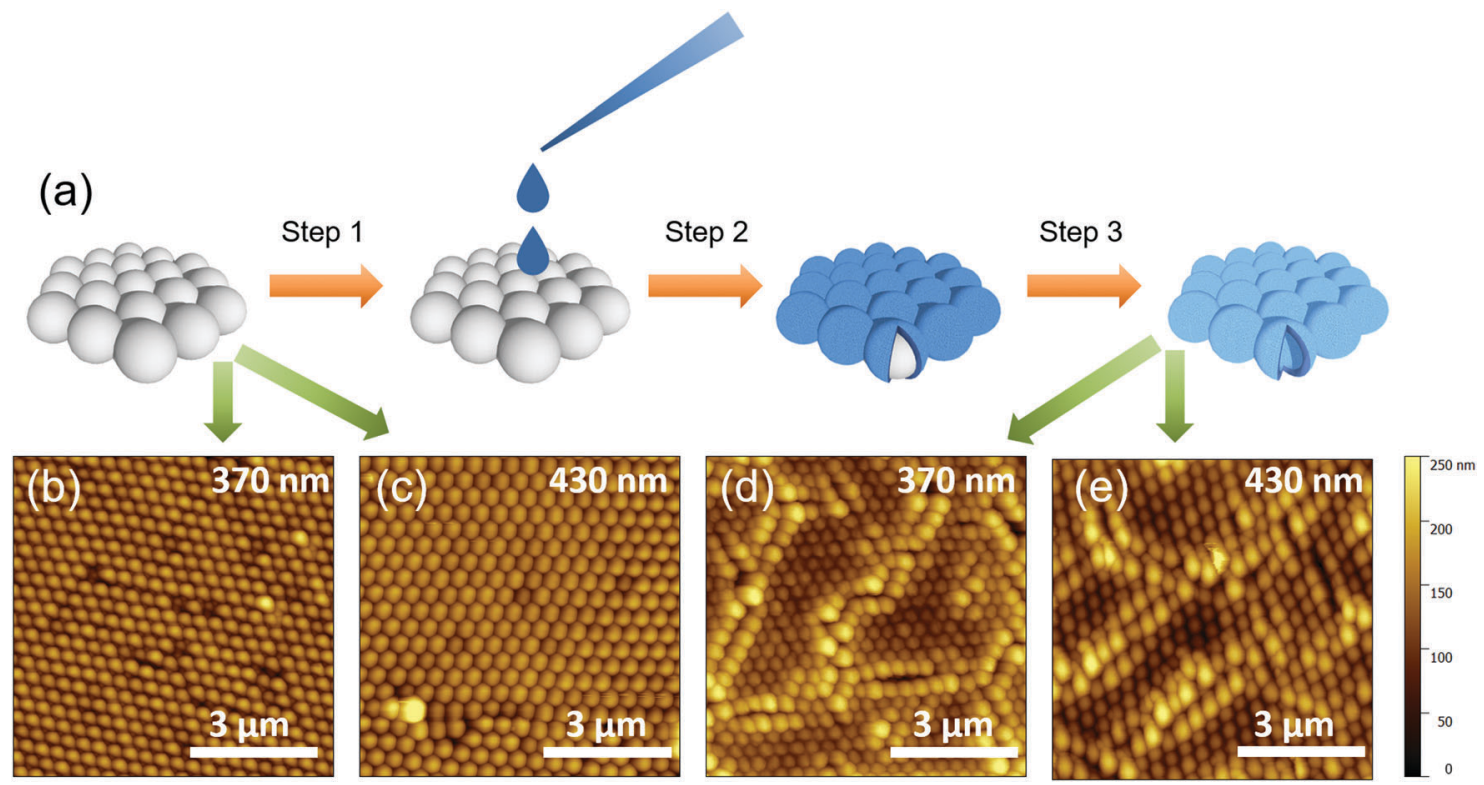

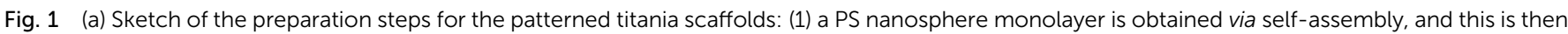

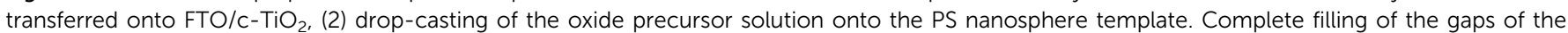

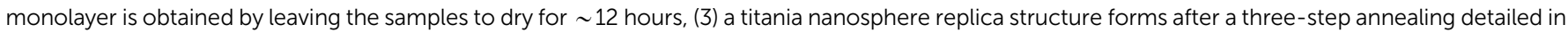

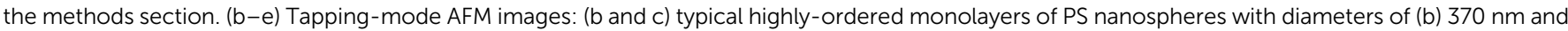

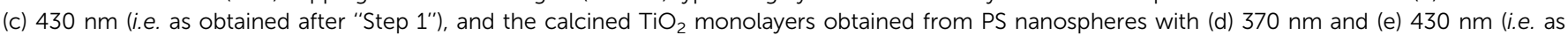
obtained after "Step 3") 
The hole-transporting material (HTM) was deposited by spincoating a solution of 2,10,7,70-tetrakis-( $N, N$-dip-methoxyphenylamine)9,92-spirobifluorene (Spiro-OmeTAD, Ossila Ltd) at $2000 \mathrm{rpm}$ for $60 \mathrm{~s}$ in a nitrogen atmosphere and left for $4 \mathrm{~h}$ in air in a closed dry box. The solution was prepared by adding $96.5 \mathrm{mg}$ of Spiro-OmeTAD, $10 \mu \mathrm{L}$ of 4-tertbutylpyridine (TBP, Aldrich) and $30 \mu \mathrm{L}$ of a $170 \mathrm{mg} \mathrm{mL}{ }^{-1} \mathrm{LiN}\left(\mathrm{CF}_{3} \mathrm{SO}_{2}\right) 2 \mathrm{M}$ solution in 1-butanol to $1 \mathrm{~mL}$ of chlorobenzene. Finally the samples were introduced into a high vacuum chamber to evaporate gold (Au) back contacts (thickness $100 \mathrm{~nm}$ ). An evaporation mask defined the device areas of both $0.15 \mathrm{~cm}^{2}$ and $0.09 \mathrm{~cm}^{2}$.

\section{Optical and morphological characterization}

The thickness of the films was measured using a surface profilometer (Dektak ${ }^{3}$ ). UV-Visible transmittance measurements were done using an Agilent 8453 spectrophotometer. Surface morphology has been investigated by using atomic force microscopy (AFM, Dimension 3100). All AFM images were recorded using tapping mode in air. FIB-SEM images have been taken by using a Carl Zeiss XB1540 “Cross-Beam” focused-ionbeam microscope, whereas ion-beam milling of the samples was carried out using Ar atoms. XPS spectra were recorded using a PHI 5000 VersaProbe II scanning XPS Microprobe ${ }^{\mathrm{TM}}$ (ULVAC-PHI, Inc.) and monochromatic $\mathrm{Al}-\mathrm{K} \alpha$ radiation $(h \nu=1486.6 \mathrm{eV})$ from an X-ray source operating at a spot size of $200 \mu \mathrm{m}$, a power of $50 \mathrm{~W}$ and an acceleration voltage of $15 \mathrm{kV}$. A surface XPS survey was conducted using a hemispherical analyser (128 channels) at a pass energy of $23.500 \mathrm{eV}$ and an energy step size of $0.200 \mathrm{eV}$; the photoelectron take off angle with respect to the surface was $45^{\circ}$; for depth profiling time sputtering was performed using an $\mathrm{Ar}^{+}$ion gun, with a $3 \times 3 \mathrm{~mm}$ raster spot, at $3 \mathrm{kV}$, with $10 \mathrm{~s}$ time steps. XRD measurements were performed using a Rigaku SmartLab diffractometer (Rigaku, Tokyo, Japan) and a $\mathrm{K} \alpha$ wavelength emitted by a $\mathrm{Cu}$ anode $(0.154 \mathrm{~nm})$ in grazing incidence configuration.

\section{Current-voltage characteristics}

Device performance was evaluated under illumination using a Class A solar simulator (ABET Technologies) at AM 1.5G and $100 \mathrm{~mW} \mathrm{~cm}^{-2}$ connected with a source-meter (Keithley2420), calibrated with a reference Silicon cell (RERASolutionsRR-1002).

\section{Results and discussion}

The fabrication method for patterning the oxide structures is sketched in Fig. 1(a). The alcoholic suspensions containing nanobeads were first dropped on the water surface, leading to the formation of a temporary alcohol layer where the beads can float and flocculate and self-assemble in well-ordered twodimensional hexagonally-packed monolayers. ${ }^{19}$ The as-formed monolayers were then transferred ${ }^{20}$ onto fluorinated tin-oxide (FTO)/compact $\mathrm{TiO}_{2}\left(\mathrm{c}-\mathrm{TiO}_{2}\right)$ coated substrates and dipped into a water reservoir. In particular, we used polystyrene (PS) nanospheres with diameters of 370 and $430 \mathrm{~nm}$, respectively (schematically shown in "step 1" in Fig. 1(a)). We then dropped the $\mathrm{TiO}_{2}$ precursor solution onto the dried monolayers (step 2), and let it slowly dry overnight (incubation). After a final calcination step in air (see the Methods section for details) the resulting structures consist of a hexagonal close-packed hollow titania microsphere monolayer (step 3).

Fig. 1(b-d) show the tapping-mode atomic force microscopy (AFM) images of the starting PS nanospheres monolayer made of nanospheres with diameters $370 \mathrm{~nm}$ (b), $430 \mathrm{~nm}$ (c), and of the corresponding titania samples obtained after the calcination step, displayed in Fig. 1(d and e) for the 370 and $430 \mathrm{~nm}$ spheres, respectively.

The titania nanosphere hexagonal close-packed structure is well reproduced from the templates (Fig. SI_1 and SI_2 (ESI $\dagger$ ) show profiles of these at higher magnification than in Fig. 1(c and d)). The obtained films are highly ordered, dense, and essentially uniform over relatively large scales (several tens of microns), even if cracks cannot be completely avoided (Fig. 1(c and d)). We also note that whereas defects in the pre-calcination samples appear to be limited to beads' "pseudovacancies", most frequently related to the presence of beads of different sizes (owing to the nanosphere polydispersity - standard deviation of the sphere size distribution $\sim 5 \%$ ), the calcination steps lead to more extended defects in the form of cracks that can be several micrometres long. We also notice that post-calcination films show enlarged "spheroids" at the border of the cracks (e.g. see Fig. 1(d)), probably because of fewer structural constraints on the growth of the templated titania films. Notably, the surface roughness of the titania spheres (Fig. SI_3a and b, ESI $\dagger$ ) is comparable to the one of the pristine polystyrene beads (Fig. SI_3a and b, ESI $\dagger$ ), and thus the optical quality is maintained.

The results of the structural and optical characterization of the nanostructured hollow nanospheres are shown in Fig. 2. The X-ray photoelectron spectroscopy (XPS) depth profile (Fig. 2(a)) confirmed the absence of residual polystyrene inside the titania nanostructures (within the $\sim 1 \%$ limits of the technique resolution). In fact, the carbon signal is above the detection threshold only at the beginning of the profiling and can be safely attributed to the ubiquitous adventitious carbon (surface impurities), since the signal disappeared after $10 \mathrm{~s}$ sputtering. Furthermore, we observe a concomitant decrease of Ti concentration with an increase of the underlying $\mathrm{Sn}$, which is present in the substrate (FTO). This confirms the formation of the $\mathrm{TiO}_{2}$ layer on top of the FTO substrate surface, and that the annealing process removed the polystyrene leaving no XPS-detectable carbon inside the oxide nanospheres. We also note that polystyrene is known ${ }^{21}$ to degrade at temperatures of $\sim 350{ }^{\circ} \mathrm{C}$, i.e. below the maximum temperature of our annealing. We also propose that the holes visible in some of the spheres might be the escape routes of the volatile degradation products during annealing.

We explored the internal structures of the spheres in the final films by cutting a small section of the films with focused ion beam (FIB) and show scanning-electron microscopy, and SEM images of these in Fig. 2(d and e). Here the beads appear to be a bit deflated due to the ion beam bombardment but, 

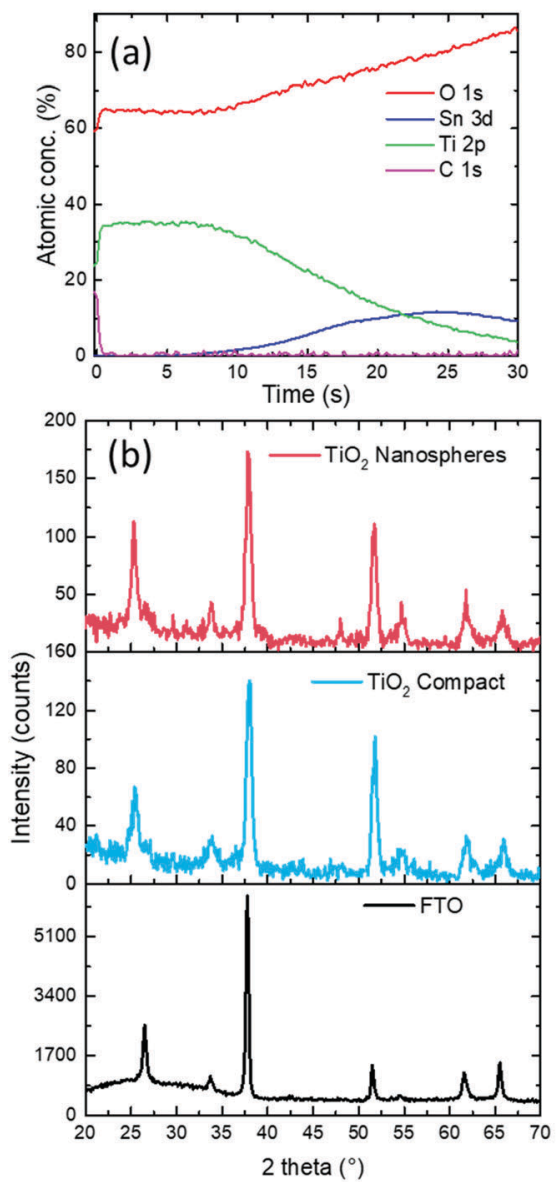
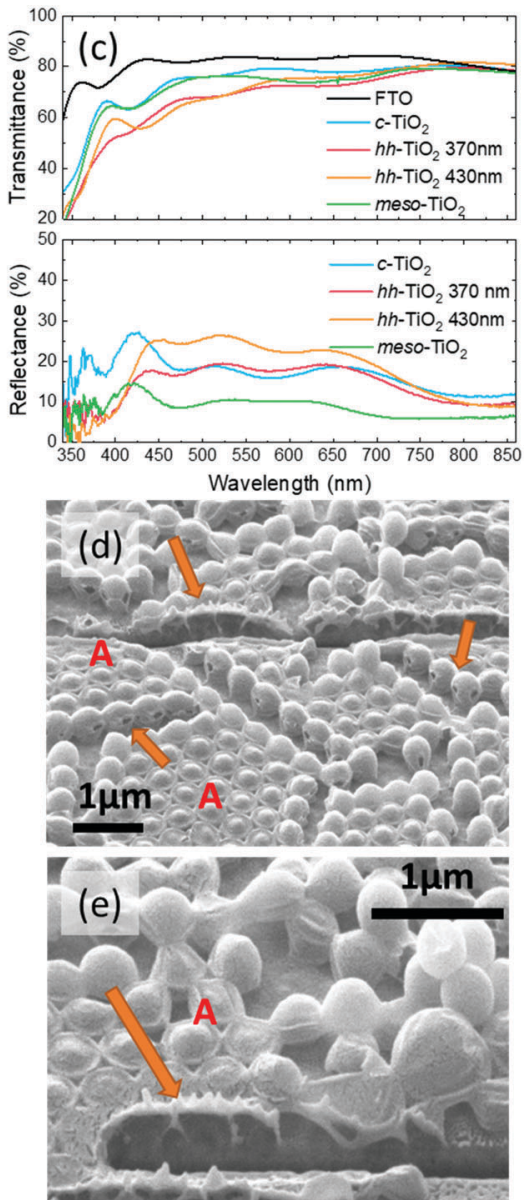

Fig. 2 (a) $\mathrm{Ar}^{+}$sputtered XPS depth profile of the titania nanosphere layer onto FTO/c- $\mathrm{TiO}_{2}$ substrates. (b) XRD spectra of the titania nanostructures and of a compact titania layer sintered onto FTO substrates and of the bare FTO. Anatase peaks of $\mathrm{TiO}_{2}: 2$ theta $=25.37^{\circ}$, fluorine-doped tin oxide (FTO) substrate peaks: 2 theta $=26.4^{\circ}, 37.6^{\circ}$. (c) Transmittance (upper panel) and reflectance (bottom panel) spectra of the titania structure synthesized onto the FTO substrates. (d) FIB-milled SEM micrograph of the titania hollow sphere monolayer. The spheres appear deflated due to the high power beam used for milling the structures. (e) Higher magnification of the milled area detailing the sphere structure confirming that they are hollow inside. In (d) and (e) the arrows indicate that the spheres are hollow inside. Interestingly many spheres feature holes of $\sim 100$ nm size mostly distributed along their equatorial plane. In some areas (e.g. the one marked " $A$ ") the spheres appear deflated if not collapsed as a result of the ion bombardment during the FIB imaging and cutting of a section to reveal the hollow structure, further confirming that they are empty shells.

more interestingly, we note that the brightness contrast indicates that the spheres are hollow (see areas indicated by arrows). Higher magnification SEM images detailing the hollow titania nanosphere wall are reported in Fig. SI_4 (ESI $\dagger$ ). Here we can observe that the thickness of the walls is around $30-50 \mathrm{~nm}$.

We also carried out X-ray diffraction (XRD) measurements to assess the crystallinity of templated films and found that the peaks are indicative of a pure anatase phase of both the titania oxide scaffold and of the compact layers (Fig. 2(b)). In these we observe in particular the presence of the peak associated with the 101 plane (at $25.37^{\circ}$ ). The other peaks are related to the FTO. XRD data were collected directly from the films on the FTO substrates.

In Fig. 2(c) we show a comparison of the transmittance and reflectance spectra of the control substrates (bare FTO, FTO/ c- $-\mathrm{TiO}_{2}$ and $\mathrm{FTO} /$ mesoporous- $\mathrm{TiO}_{2}\left(\right.$ meso- $\left.\left.\mathrm{TiO}_{2}\right)\right)$ and substrates with the nanopatterned $\mathrm{TiO}_{2}$ structures made from the microsphere monolayers. Whereas the $\mathrm{c}-\mathrm{TiO}_{2}$ and the meso- $\mathrm{TiO}_{2}$ films simply reduce the transmittance, compared to the FTO sample, the nanopatterned structures show considerably different characteristics. In fact, in the transmittance spectra we observe the presence of many dips at wavelengths different from those of the $\mathrm{c}-\mathrm{TiO}_{2}$ layer. The origin of such a spectral structure is related to light scattering induced by the monolayer. ${ }^{22}$ In particular, the $370 \mathrm{~nm}$ sphere $\mathrm{hh}-\mathrm{TiO}_{2}$ sample shows a mean reflectance comparable to that of the $\mathrm{c}^{-\mathrm{TiO}_{2}}$ one (for $\lambda>450 \mathrm{~nm}$ or so), but a lowered mean transmittance. This is interesting as the absorbance by titania is expected to be comparable in both cases (based on the amount and optical quality of the material, also as shown in SEM observations of the FIB cross-section), thereby implying an increased scattering of the light propagating beyond the very first layers of the templated titania.

Clearly, the porosity of these structures, which is demonstrated by the figures above, is directly linked to the sphere size. This is particularly important for perovskite-based solar cells in 
which adequate filling of pores is a crucial parameter for ensuring intimate electrical contact between the titania and the active layer, and thus for optimizing the final device performance, ${ }^{23,24}$ especially considering the tendency of the perovskites to form large crystals. ${ }^{25}$ We note that although the presence of smaller crystals has been demonstrated to be disadvantageous in terms of charge mobility, due to an increased number of grain boundaries, ${ }^{26}$ having uniform crystal domains with small grain sizes might improve the interfacial contact between the perovskite and the $\mathrm{TiO}_{2}$ and an overall improvement of the device properties is expected where the interfacial impedance provides the dominant contribution to the series resistance of the solar cell.

In our structures, analyses of the AFM images show that the perovskite films on $\mathrm{hh}-\mathrm{TiO}_{2}$ (Fig. SI_5a, ESI $\dagger$ ) appear to be formed of small domains, with a root mean square (RMS) roughness of $47.3 \pm 0.9 \mathrm{~nm}$, whereas the films on top of the compact $\mathrm{TiO}_{2}$ (Fig. SI_5b, ESI $\dagger$ ) layer show larger perovskite crystalline domains, with an RMS roughness of $51.4 \pm 1.6 \mathrm{~nm}$. The film prepared onto meso-TiO 2 (Fig. SI_5c, ESI $\dagger$ ) appears to be formed by relatively large crystals, similar to the film prepared onto $\mathrm{c}-\mathrm{TiO}_{2}(\mathrm{RMS}=57.6 \pm 3.1 \mathrm{~nm})$, but in addition the perovskite layer appears less continuous and not fully infiltrated into the titania as suggested by the holes in the perovskite films and the titania underneath, as indicated by the arrows. We can gain further insights by looking at the crystallinity of the perovskite films prepared onto the different substrates (Fig. SI_6, ESI $\dagger$ ). Although the XRD peak positions are the same $\left(2 \theta=14.2^{\circ}\right.$ and $28.4^{\circ}$, respectively), their widths are slightly broader for the perovskites grown on the nanospheres, with a full width at half maximum, FWHM, increasing from $0.227^{\circ}$ for the perovskite prepared on the $\mathrm{c}-\mathrm{TiO}_{2}$ layer to $0.339^{\circ}$ for the film prepared onto the meso- $\mathrm{TiO}_{2}$ scaffold, and to $0.236^{\circ}$ and $0.234^{\circ}$ for the ones prepared on hh- $\mathrm{TiO}_{2}$ (starting from 370 and $430 \mathrm{~nm}$ diameter beads respectively). This confirms that the crystal domain size is the smallest for films on meso- $\mathrm{TiO}_{2}$ and maximum in the case of $\mathrm{c}-\mathrm{TiO}_{2}$, consistent with the AFM images.

With potential applications to solar cells in mind, we incorporated the films described above into solar cells obtained by capping the perovskite layers with spiro-OmeTAD $\left(2,2^{\prime}, 7,7^{\prime}\right.$ tetrakis[ $N, N$-di(4-methoxyphenyl)amino]-9,9'-spirobifluorene), an organic hole-conductor, and a gold top electrode. We also compared these devices with cells incorporating a mesoporous and a compact titania layer (schematically illustrated in Fig. 3(a)), as typically used in the preparation of perovskite solar cells. ${ }^{27}$

The $J-V$ characteristics of illustrative examples of the different types of devices are shown in Fig. 3(b), whereas Fig. 3(c) provides full details of the typical device performance parameters (power conversion efficiency, PCE, fill-factor, FF, open-circuit voltage, $V_{\mathrm{oc}}$, and short circuit current $J_{\mathrm{sc}}$ ) as extracted from the characteristics of four working devices for each structure.
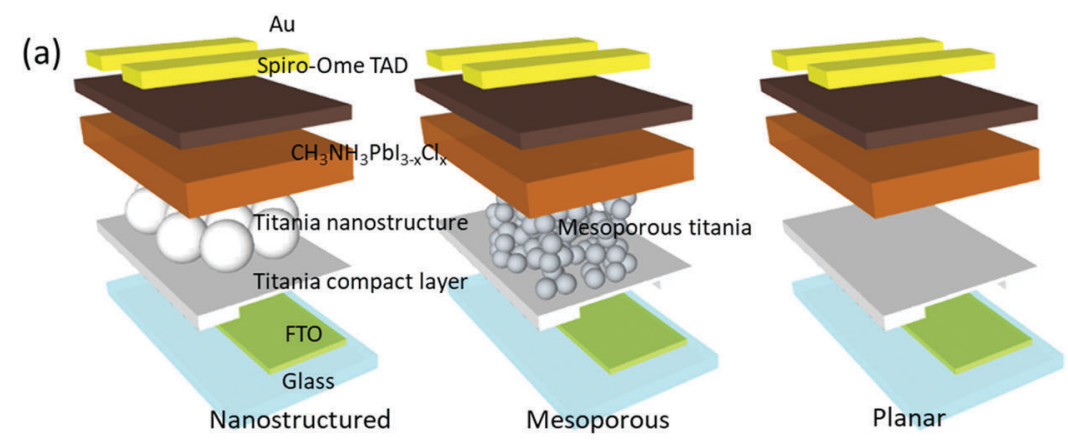

(b)

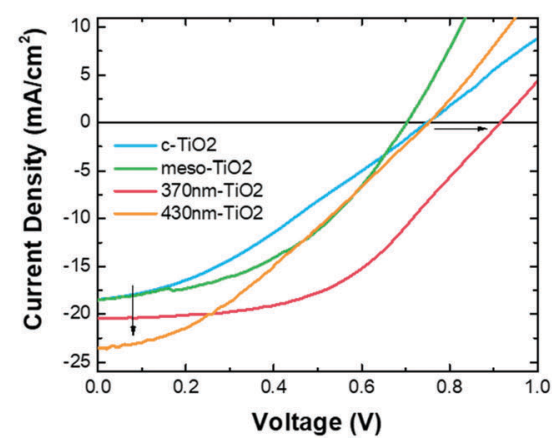

(c)

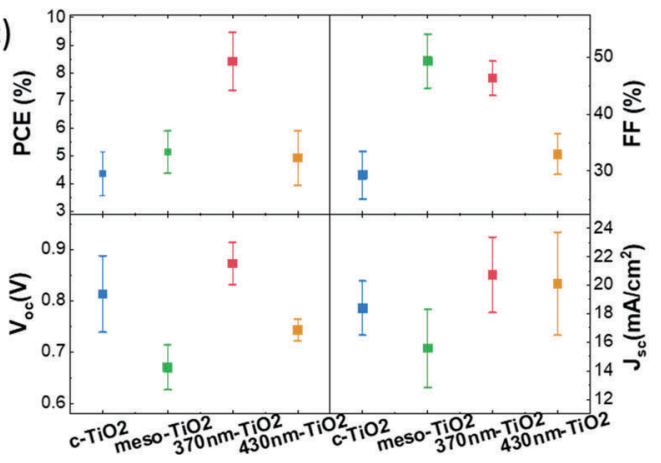

Fig. 3 (a) Scheme of the device prepared with the ordered nanostructure, with the "standard" titania mesoporous scaffold, and with the perovskite coated on top of the compact titania layer. ( $b$ and c) Electrical characterization and device performance analysis: (b) $J-V$ curves of the devices under illumination. The corresponding $J-V$ curves in the dark are reported in Fig. SI_7 (ESI $\dagger$ ). The arrows highlight the enhanced $J_{\text {sc }}$ and $V_{\text {oc }}$ obtained for the cells prepared with the nanostructured titania layer. (c) Device performance parameters: power conversion efficiency (PCE), fill factor (FF), open circuit voltage $\left(V_{o c}\right)$, and short-circuit current density $\left(J_{s c}\right)$. 
The most prominent feature that emerges from inspection of this data is the distinctively larger value of the power conversion efficiency (PCE) of the $370 \mathrm{~nm}$ sphere cells compared to all others i.e. $\sim 8.5 \pm 1 \%$, albeit we do note that these are below the state-of-the-art values reported for $\mathrm{c}-\mathrm{TiO}_{2}$ or meso-TiO ${ }_{2}$ cells. $^{28,29}$ We attribute this lower PCE to the nonoptimized perovskite growth process (most likely related to the moisture air level during casting of the active layer, and calibration of the annealing temperature). The interesting and appealing aspect however is that the hh- $\mathrm{TiO}_{2}(370 \mathrm{~nm}$ sphere) cells are not as sensitive to these parameters. The question arises as to why the $430 \mathrm{~nm}$ sphere cells do not show the same level of PCE, and to get a better insight into this, we need to look into more details of the other individual parameters. Starting with the $V_{\text {oc }}$, we note an enhanced average value in $\mathrm{hh}-\mathrm{TiO}_{2}(370 \mathrm{~nm})$ compared to all other cells, even if c-TiO ${ }_{2}$ shows a relatively large standard deviation. We can rule out that the enhancement for $\mathrm{hh}-\mathrm{TiO}_{2}(370 \mathrm{~nm})$ is due to a significant change of the work function, as Kelvin probe measurements (Fig. SI_7, ESI $\dagger$ ) indicated similar values. In fact, the values obtained from hh- $\mathrm{TiO}_{2}(\sim 4.49 \mathrm{eV})$ are comparable to that of the mesoporous one $(4.45 \mathrm{eV})$ and only slightly higher than that of the $\mathrm{c}-\mathrm{TiO}_{2}$ one $(4.2 \mathrm{eV})$.

Alternatively the observed increase in $V_{\text {oc }}$ for hh- $\mathrm{TiO}_{2}$ could be explained by the suppression of shunts between the holeblocking layer $\left(\mathrm{c}-\mathrm{TiO}_{2}\right)$ and the hole-transport layer (SpiroOmeTAD), thanks to the improved isolation in turn induced by the ordered microstructures with a controlled and optimized thickness. In particular, such an enhancement was observed only for the scaffold prepared from the smaller nanospheres $(370 \mathrm{~nm})$. This is in agreement with our expectations that any defects generated by a missing (or exploded) bead would generate a bigger impact on the device properties for the $430 \mathrm{~nm}$ sphere film. Similarly we expect the cracks to be larger, and therefore provide more significant shunt channels in the hh- $\mathrm{TiO}_{2}$ devices.

Interestingly, we note that the average short-circuit current densities $\left(J_{\mathrm{sc}}\right)$ are also higher for the nanostructured devices than for the devices prepared on both the compact titania layer and on the "standard" device prepared onto the nanostructured titania. We attribute this to two facts: first, as confirmed by the XRD pattern and AFM, the perovskite crystalline domains are smaller than the ones obtained onto the compact titania, and for this reason they are in full contact with the electron-transporting layer; second, the ordered layer is a better scattering medium compared the other two, and this improves light harvesting in the active layer. The current voltage curves presented here are scanned from forward bias to short-circuit, i.e. in the reverse condition. We observed hysteresis in the $J-V$ curves when measuring in forward bias, with PCE lower than that determined from the current voltage curves measured in the reverse condition. ${ }^{30,31}$ For instance in Fig. SI_9 (ESI $\dagger$ ) we show the forward current voltage scans for a device prepared onto the nanostructured titania, which exhibited a $J-V$ derived PCE of $7.3 \%$ in reverse bias with a PCE of just over $4.6 \%$ in forward bias. Remarkably, a further measurement from forward bias to short-circuit showed the same behavior (and PCE) of the first measurement.

Series resistance $\left(R_{\mathrm{S}}\right)$ and shunt resistance $\left(R_{\mathrm{SH}}\right)$ for devices have been obtained by fitting the $J-V$ curves with a one-diode model (Fig. SI_10, ESI $\dagger)^{32}$ and the mean values are reported in Fig. SI_10 (ESI $\dagger$ ). Higher shunt resistances have been found for both the nanostructured and mesoporous titania confirming that the recombination paths are significantly blocked in the microstructured titania devices, thanks to the better isolating lateral layer, compared to the just compact titania layer. Furthermore, the lower series resistances found in the nanostructured titania confirm that there is an improved movement of current through the device, in good agreement with a better morphology of the perovskite layers. ${ }^{33}$

\section{Conclusion}

In conclusion, we have presented a low-cost, scalable technique for fabricating a highly ordered patterned titanium oxide photonic scaffold. Illustrative applications of such scaffolds to perovskite solar cells prepared onto this scaffold show that these cells exhibit efficiencies of about 9\%, higher than unoptimized "reference" cells prepared in our laboratory with mesoporous and compact titania layers. We attribute this enhancement to a combination of increased light-trapping inside the perovskite active layer induced by the feedback structure and the formation of an improved perovskite film, together with an improved film formation process, leading to a smaller crystal size, and a better electrical contact between the perovskite active materials and the titania electrodes. In particular, we propose that, thanks to the controlled and uniform thickness of the titania scaffold that can be obtained with these layers, shunts are reduced and/or suppressed, thereby affording higher device efficiency compared to our unoptimized reference cells.

\section{Conflicts of interest}

There are no conflicts of interest to declare.

\section{Acknowledgements}

We thank professor Henry Snaith and his group for valuable discussion and technical assistance. This work was supported by the EU H2020 ETN SYNCHRONICS under grant agreement 643238 , the EU H2020 ITN project iSwitch under grant agreement 642196, PON02_00153_2939517 TESEO. GMP was supported by an IMPACT PhD studentship co-sponsored by UCL and ISIS-Neutron and Muon Facility (Science and Technology Facilities Council). MD was supported by FP7 European Project GONEXTs (Contract 309201). FC is a Royal Society Wolfson Research Merit Award holder. Underlying research data can be accessed via the UCL Research Publications Service (RPS) http://rps.ucl.ac.uk and searching for c7tc04070a. 


\section{References}

1 D. O. Scanlon, C. W. Dunnill, J. Buckeridge, S. A. Shevlin, A. J. Logsdail, S. M. Woodley, C. R. A. Catlow, M. J. Powell, R. G. Palgrave, I. P. Parkin, G. W. Watson, T. W. Keal, P. Sherwood, A. Walsh and A. A. Sokol, Nat. Mater., 2013, 12, 798-801.

2 B. O'Regan and M. Gratzel, Nature, 1991, 353, 737-740.

3 M. M. Lee, J. Teuscher, T. Miyasaka, T. N. Murakami and H. J. Snaith, Science, 2012, 338, 643-647.

4 A. Fujishima and K. Honda, Nature, 1972, 238, 37-38.

5 D. Bi, S.-J. Moon, L. Haggman, G. Boschloo, L. Yang, E. M. J. Johansson, M. K. Nazeeruddin, M. Grätzel and A. Hagfeldt, RSC Adv., 2013, 3, 18762-18766.

6 X. Yu, S. Chen, K. Yan, X. Cai, H. Hu, M. Peng, B. Chen, B. Dong, X. Gao and D. Zou, J. Power Sources, 2016, 325, 534-540.

7 N. Cheng, P. Liu, S. Bai, Z. Yu, W. Liu, S.-S. Guo and X.-Z. Zhao, J. Power Sources, 2016, 321, 71-75.

8 S. Guarnera, A. Abate, W. Zhang, J. M. Foster, G. Richardson, A. Petrozza and H. J. Snaith, J. Phys. Chem. Lett., 2015, 6, 432-437.

9 M. He, D. Zheng, M. Wang, C. Lin and Z. Lin, J. Mater. Chem. A, 2014, 2, 5994-6003.

10 M. T. Horantner, W. Zhang, M. Saliba, K. Wojciechowski and H. J. Snaith, Energy Environ. Sci., 2015, 8, 2041-2047.

11 X. Chen, S. Yang, Y. C. Zheng, Y. Chen, Y. Hou, X. H. Yang and H. G. Yang, Adv. Sci., 2015, 2, 1500105.

12 M. Iida, T. Sasaki and M. Watanabe, Chem. Mater., 1998, 10, 3780-3782.

13 J.-M. Wu, X.-M. Song and M. Yan, J. Hazard. Mater., 2011, 194, 338-344.

14 X. Gong, L. Wang and W. Wen, Chem. Commun., 2009, 4690-4692, DOI: 10.1039/B908932B.

15 A. M. Collins, C. Spickermann and S. Mann, J. Mater. Chem., 2003, 13, 1112-1114.

16 T. Nakashima and N. Kimizuka, J. Am. Chem. Soc., 2003, 125, 6386-6387.

17 J. Yun, J. Ryu, J. Lee, H. Yu and J. Jang, J. Mater. Chem. A, 2016, 4, 1306-1311.

18 T. M. Brown, G. M. Lazzerini, L. J. Parrott, V. Bodrozic, L. Bürgi and F. Cacialli, Org. Electron., 2011, 12, 623-633.
19 G. D. Moon, T. I. Lee, B. Kim, G. Chae, J. Kim, S. Kim, J.-M. Myoung and U. Jeong, ACS Nano, 2011, 5, 8600-8612.

20 V. Robbiano, M. Giordano, C. Martella, F. D. Stasio, D. Chiappe, F. B. de Mongeot and D. Comoretto, Adv. Opt. Mater., 2013, 1, 389-396.

21 J. D. Peterson, S. Vyazovkin and C. A. Wight, Macromol. Chem. Phys., 2001, 202, 775-784.

22 A. Belardini, A. Benedetti, M. Centini, G. Leahu, F. Mura, S. Sennato, C. Sibilia, V. Robbiano, M. C. Giordano, C. Martella, D. Comoretto and F. B. de Mongeot, Adv. Opt. Mater., 2014, 2, 208-213.

23 J. J. Choi, X. Yang, Z. M. Norman, S. J. L. Billinge and J. S. Owen, Nano Lett., 2014, 14, 127-133.

24 O. E. Semonin, G. A. Elbaz, D. B. Straus, T. D. Hull, D. W. Paley, A. M. van der Zande, J. C. Hone, I. Kymissis, C. R. Kagan, X. Roy and J. S. Owen, J. Phys. Lett., 2016, 7, 3510-3518.

25 V. M. Burlakov, G. E. Eperon, H. J. Snaith, S. J. Chapman and A. Goriely, Appl. Phys. Lett., 2014, 104, 091602.

26 S. Mastroianni, F. D. Heinz, J. H. Im, W. Veurman, M. Padilla, M. C. Schubert, U. Wurfel, M. Gratzel, N. G. Park and A. Hinsch, Nanoscale, 2015, 7, 19653-19662.

27 M. A. Green, A. Ho-Baillie and H. J. Snaith, Nat. Photonics, 2014, 8, 506-514.

28 J. Burschka, N. Pellet, S.-J. Moon, R. Humphry-Baker, P. Gao, M. K. Nazeeruddin and M. Gratzel, Nature, 2013, 499, 316-319.

29 P. Qin, M. Paulose, M. I. Dar, T. Moehl, N. Arora, P. Gao, O. K. Varghese, M. Grätzel and M. K. Nazeeruddin, Small, 2015, 11, 5533-5539.

30 H. J. Snaith, A. Abate, J. M. Ball, G. E. Eperon, T. Leijtens, N. K. Noel, S. D. Stranks, J. T.-W. Wang, K. Wojciechowski and W. Zhang, J. Phys. Lett., 2014, 5, 1511-1515.

31 E. L. Unger, E. T. Hoke, C. D. Bailie, W. H. Nguyen, A. R. Bowring, T. Heumuller, M. G. Christoforo and M. D. McGehee, Energy Environ. Sci., 2014, 7, 3690-3698.

32 K. Miyano, N. Tripathi, M. Yanagida and Y. Shirai, Acc. Chem. Res., 2016, 49, 303-310.

33 A. Listorti, E. J. Juarez-Perez, C. Frontera, V. Roiati, L. Garcia-Andrade, S. Colella, A. Rizzo, P. Ortiz and I. Mora-Sero, J. Phys. Chem. Lett., 2015, 6, 1628-1637. 